\title{
Macroglossia due to Systemic Amyloidosis: Is There a Role for Radiotherapy?
}

\author{
Isabelle Thibault Isabelle Vallières
}

Département de Radio-oncologie, L'Hôtel-Dieu de Québec, Centre Hospitalier

Universitaire de Québec (CHUQ), Québec, Qué., Canada

\section{Key Words}

Amyloidosis $\cdot$ Macroglossia $\cdot$ Radiation $\cdot$ Tongue

\begin{abstract}
Background: Macroglossia due to amyloid depositions can cause cosmetic problems and functional disability, and can lead to life-threatening airway obstruction. Management of macroglossia in systemic amyloidosis is controversial, and the role of surgery is unclear. Case Description: We present a case of a 66-year-old woman affected by macroglossia due to light chain amyloidosis who presented with eating and breathing difficulties. Because of prior successful results of radiotherapy for localized amyloid disease, our patient was treated with external beam radiation therapy (20 Gy in 10 fractions). The treatment was well tolerated by the patient. However, her systemic amyloidosis progressed, with a subclinical increase in tongue width.
\end{abstract}

Conclusions: This is the first reported use of radiotherapy for amyloidosis of the tongue. There was no evidence of benefit using a total dose of $20 \mathrm{~Gy}$. This therapeutic modality is not recommended for the routine management of macroglossia.

\section{Introduction}

Amyloidosis is a group of disorders characterized by extracellular deposition of insoluble amyloid fibrils within tissues. This deposition of amyloid can be either localized or systemic. The most common form of systemic disease is light chain (AL) amyloidosis, which results from the deposition of monoclonal immunoglobulin light chains.

Amyloid involvement of the tongue is almost always secondary to systemic AL amyloidosis and can occur in up to $40 \%$ of cases [1-5]. Serious respiratory, eating and speech problems can be caused by a lingual involvement. Treatment of this condition is controversial and challenging. 
We present a case of an elderly woman suffering from systemic AL amyloidosis with severe macroglossia. To our knowledge, this is the first case report that describes the use of external beam radiation therapy (EBRT) as treatment for macroglossia due to amyloidosis.

\section{Case Report}

A 66-year-old woman was seen in our radiation oncology department for symptomatic progressive macroglossia 7 years after her initial diagnosis of amyloidosis. The patient was a non-smoker, nondrinker and had no significant family history. Her past medical history was notable for a hepatitis A infection and a latent tuberculosis infection treated with isoniazid. Her past surgical history included a hysterectomy and a bilateral ovariectomy. She also underwent simultaneous bilateral carpal tunnel surgery twice (between 1998 and 2001) before the diagnosis of systemic AL amyloidosis was made after two successive positive tongue biopsies. Amyloid deposits were also identified on bone marrow examination and were present in bone as well as in articular tissues of both shoulders, as suggested by magnetic resonance imaging. Further tests revealed a bone marrow plasmacytosis of between 5 and $10 \%$, a small decrease in serum gamma globulins and a kappa light chain band on serum immunofixation.

The patient was initially treated with autologous stem cell transplantation (SCT), but her response to treatment was limited. Approximately 1 year later, she complained of dyspnea and developed obstructive sleep apnea requiring nasal continuous positive airway pressure (CPAP). Her respiratory symptoms were primarily related to the progressive macroglossia. The patient was later hospitalized for mild pulmonary hypertension and right heart failure. Besides sleep apnea, other possible explanations for the development of pulmonary hypertension included cardiac amyloidosis, although not all criteria for cardiac amyloid were met. The patient also received a pacemaker implant for sinus node dysfunction.

A variety of systemic treatment regimens were used to control the progression of her disease following SCT. Medications given included etanercept and thalidomide; however, no response could be obtained. Unfortunately, after receiving thalidomide, the patient developed bilateral deep vein thrombosis, and a combination of oral melphalan and dexamethasone was prescribed for 2 years until the patient developed an osteoporotic vertebral compression fracture.

In January 2009, the patient presented again with progressive macroglossia causing speech, mastication, swallowing and breathing difficulties. She experienced upper dysphagia when swallowing some solids, such as rice and pills. She also reported a grade 2 dyspnea and was still using her CPAP for sleep apnea. Physical examination revealed an oral opening of $<4 \mathrm{~cm}$ and a markedly enlarged tongue, from its base to its tip, with a maximum tongue width of $6 \mathrm{~cm}$. Indentations caused by her teeth were also present on the tongue (fig. 1). Except for a significant submandibular gland enlargement, the rest of the physical examination was within normal limits.

Since the patient had already received several different treatments, further therapeutic options were explored for the management of her macroglossia. Surgery was excluded due to her cardiopulmonary comorbidities. After review of the literature, we proposed using EBRT as palliative therapy for her macroglossia ( $\underline{\text { fig. }}$. $)$ because of its documented success in the treatment of the localized form of amyloidosis. A total dose of 20 Gy was delivered in 10 fractions using a 6-MV linear accelerator. Treatment was given 5 days a week at 2 Gy per fraction, over a period of 14 days. Before the first treatment, the patient was evaluated by a cardiologist due to her pacemaker status. During treatment, telemetry monitoring and an appropriate shielding block were used to prevent pacemaker dysfunction.

The patient was followed up at 1-month intervals for the first 3 months after radiotherapy, and then every $2-4$ months. The primary aim of this study was to assess the tongue size. The secondary end point was the evaluation of the symptomatic response, respiratory and deglutition functions, mouth opening and side effects.

After radiotherapy, the patient developed acute grade 1 stomatitis at the base and dorsum of the tongue, grade 2 stomatitis on the indented lateral margins of the tongue, grade 1 dysgeusia and grade 2 pain, according to the National Cancer Institute Common Terminology Criteria for Adverse Events 
(NCI CTCAE, version 3.0). No radiation-induced mucositis was observed on her cheeks or palate, and no upper respiratory toxicity occurred. A slight improvement in her sleep apnea and a reduction in CPAP from 15 to $12 \mathrm{~cm} \mathrm{H}_{2} \mathrm{O}$ were observed. Unfortunately, the systemic amyloidosis subsequently progressed. The patient presented with subcutaneous amyloid nodules, increased arthralgia and general deterioration. At the 8-month follow-up, her tongue width was $7 \mathrm{~cm}$ while the tongue indentations appeared unchanged. Mouth opening, eating ability as well as speech and respiratory symptoms remained stable. No late toxicity was observed. At the 10-month follow-up, our patient reported a subjective clinical amelioration of her macroglossia, even though no significant amelioration of objective signs could be observed. The patient later experienced progression of systemic amyloidosis, severe heart failure and loss of autonomy. She died of multilobar pneumonia after 14 months of follow-up.

\section{Discussion}

Amyloidosis is a generic term for a heterogeneous group of diseases associated with extracellular tissue deposits of fibril proteins sharing a similar structure. Classically, identification of amyloid deposits is based on the presence of an apple-green birefringence under polarized light microscopy after staining with Congo red dye. There are several forms of amyloidosis, and its modern classification depends on the nature of the protein precursor of the amyloid fibrils. To date, 25 different human protein precursors have been identified [6]. The amyloid protein AL is derived from monoclonal lambda or kappa immunoglobulin light chains, the commonest form of clinical amyloidosis in developed countries [2, 5,7]. Distribution of AL amyloid deposits can be systemic or, more rarely, restricted to one organ or tissue. The reported incidence of systemic AL amyloidosis, previously known as primary amyloidosis, is 8 per million patients per year $[3,5]$. This systemic disorder coexists with multiple myeloma in approximately $10 \%$ of cases.

Amyloidosis affecting the head and neck is rare, and most cases have the AL type of amyloid $[5,6]$. Any site may be involved; however, the most common sites are the larynx and the tongue. Amyloidosis of the head and neck usually presents as a localized lesion, except in cases with an involvement of the tongue, which is typically secondary to the systemic disease $[3,5,8-10]$. Tongue involvement manifests as a firm to rubbery macroglossia; less frequently, it presents as a yellowish nodule or a raised white lesion [8]. Some authors consider macroglossia as a paraneoplastic manifestation of plasma cell dyscrasia which is virtually diagnostic of AL amyloidosis when associated with dominant organ dysfunction $[2,8]$. Submandibular gland enlargement also frequently presents with macroglossia in AL amyloidosis $[8,11]$.

Macroglossia eventually causes cosmetic and functional problems. Speech impairment, mastication and swallowing problems, breathing and sleeping difficulties as well as lifethreatening complications such as airway obstruction may occur. Management of macroglossia depends primarily on the extent of organ involvement, on whether amyloidosis is localized or systemic, and on the amyloid protein type (different therapies exist for different types of amyloid) [7].

While it is well accepted that the localized form of lingual amyloidosis can be reasonably treated with surgery $[2,9,12,13]$, no established treatment exists for the management of macroglossia due to systemic amyloidosis. Cases reported in the literature are rare, and scientific evidence is lacking. The treatment options, gathered from 
published French- and English-language articles, can be classified into systemic therapy, surgical intervention and surgical bypass measures.

The current treatment for systemic AL disease is based on a systemic therapy which aims to suppress the plasma cell clone producing the amyloidogenic immunoglobulin light chain component. Standard treatment consists of high-dose melphalan and autologous SCT for selected patients, or a combination of melphalan and dexamethasone [14-16]. However, two-thirds of newly diagnosed patients are not eligible for SCT [7], and chemotherapy administration may be limited by the patient's inability to tolerate the side effects, especially in older patients. Despite recent advances, the hematologic response to systemic therapy is still often incomplete, delayed or absent. Furthermore, organ damage such as macroglossia may be irreversible in spite of a hematologic response. Supportive care is thus essential to preserve function and maintain the patient's quality of life $[2,5,17]$.

As local therapy for macroglossia, the role of surgery remains unclear because of the poor long-term prognosis and the morbidity associated with the surgical procedure. Contraindications to surgical treatment include difficult intubation, abnormal bleeding tendency and difficulty in suturing and wound healing due to amyloidosis. Moreover, recurrence of amyloid depositions is frequent. Therefore, tracheostomy and gastrostomy or jejunostomy for enteral alimentation are the only approaches available for the majority of patients affected by systemic amyloidosis $[1,5]$.

The role of radiation therapy in the management of macroglossia secondary to amyloid has not been reported. To our knowledge, this is the first published case report on this treatment.

The rationale behind the use of EBRT is its effect on the clonal plasma cells responsible for producing the light chains and amyloid fibrils $[18,19]$. Plasma cells are radiosensitive and their response to radiotherapy has been demonstrated in multiple myeloma and plasmocytoma [20]. In addition, successful results have been obtained with EBRT for the radical or postoperative therapy of tracheobronchial amyloidosis [18, 20-24], localized laryngeal amyloidosis [25] and localized periocular or orbital amyloidosis [19, 26-28]. However, this therapy of the localized form of the disease has been questioned by some scientists due to the scarcity of plasma cells in a number of localized cases, especially in tracheobronchial amyloidosis. In localized amyloidosis, a clone of plasma cells is usually present at the site of amyloid deposition and has been implicated in the synthesis of large amounts of amyloid precursors [18]. Nevertheless, other researchers believe that radiotherapy operates via other mechanisms including its effect on local vasculature versus an induction of an immune response [20].

In their series, Neben-Wittich et al. [20] presented 7 consecutive patients treated with radical EBRT for tracheobronchial AL amyloidosis. All patients showed a favorable response to treatment, with improvement or stabilization of their symptoms. For upper and lower respiratory tract involvement, Gallivan and Gallivan [25] reported that 8 patients out of 10 affected by laryngeal or tracheobronchial amyloid deposition achieved stabilization of their disease with $20 \mathrm{~Gy}$.

More recently, Oluk and Murphy [29] noted a significant regression of nasal and nasopharyngeal amyloidosis with the use of radiotherapy in a patient affected by AL amyloidosis associated with multiple myeloma. Improvement in symptoms also occurred. 
The current case is the first reported use of radiotherapy as treatment for macroglossia due to systemic AL amyloidosis. Overall, EBRT was well tolerated but failed to provide significant palliation of symptoms. Furthermore, a slight increase in tongue width occurred after radiation therapy.

If progression of amyloidosis is considered as the most probable cause of macroglossia deterioration in our patient, we must assess the efficacy of the administered treatment with regard to the dose-response relationship. An insufficient dose may explain the failure of radiotherapy in our case because we favored a safer dose administration in an attempt to prevent edema formation. Thus, only $20 \mathrm{~Gy}$ were given. In comparison, in the reported literature, doses of 30-34 Gy have been delivered for localized orbital amyloidosis [26, 28] and doses of 20-24 Gy for tracheobronchial amyloidosis [20, 24, 30]. Our treatment using $20 \mathrm{~Gy}$ may have been insufficient for a case with tongue involvement. On the other hand, failure of EBRT in our patient may be partially explained by the progression of her systemic amyloidosis.

Alternatively, if we entertain the notion that the increase in tongue width was secondary to a radiation-induced side effect, it would be reasonable to expect resolution of hyperemia and edema over time, as well as progressive fibrosis and atrophy. However, these changes were not observed in our patient after 14 months of follow-up.

Although EBRT has been shown to be effective in the treatment of specific forms of amyloidosis involving the respiratory tract and head and neck, our clinical outcome does not support the use of EBRT for macroglossia in patients with AL amyloidosis. It is important to consider factors such as evolution of systemic amyloidosis, previous treatment received, comorbidities, airway and swallowing status, patient preference and dose of EBRT delivered. Also, it is essential to analyze dose-response relationships for efficacy and safety. Because there are relatively few systemic AL amyloidosis patients with severe macroglossia and because of the multitude of factors to consider, it would be difficult to perform randomized trials in this setting. Accordingly, all case reports are important in order to define the best therapeutic strategy for these patients.

\section{Conclusions}

Radiation therapy is not recommended for the standard management of macroglossia due to systemic AL amyloidosis. There was no evidence of a therapeutic benefit with 20Gy irradiation on a short-term basis.

\section{Disclosure Statement}

All authors declare that no financial conflicts of interest exist. 

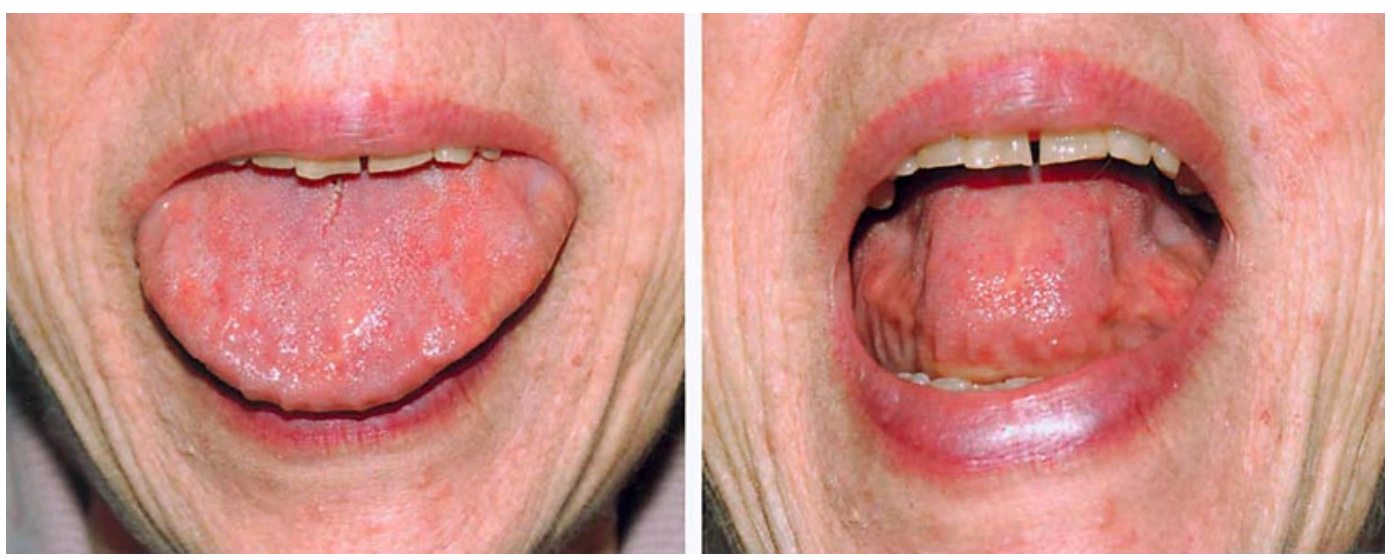

Fig. 1. Oral examination reveals enlargement of the tongue, scalloping at the edge of the tongue and deep indentations adjacent to the premolars and molars. Due to the constant lingual pressure exerted by the teeth, the patient experienced oral discomfort. 


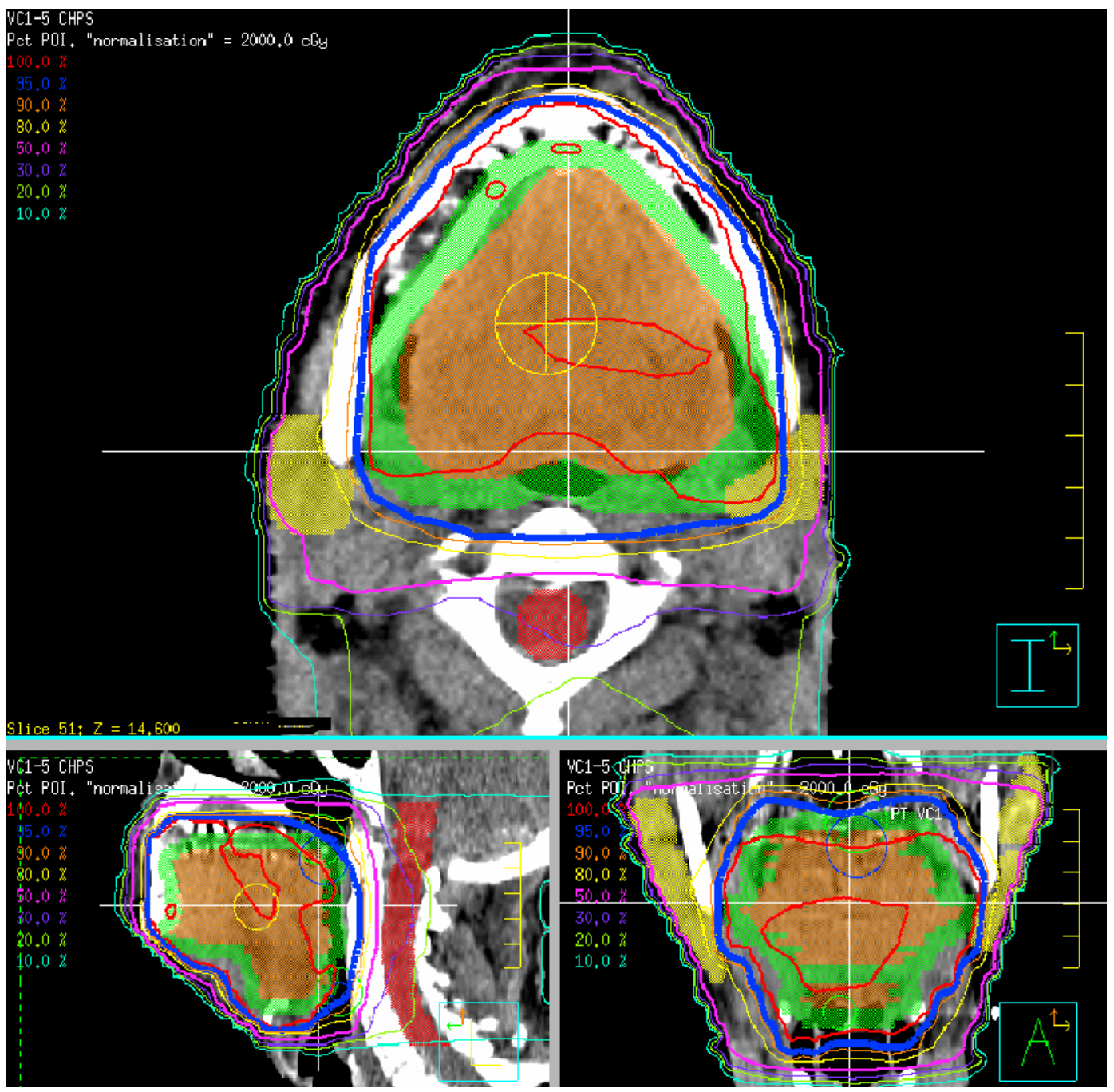

Fig. 2. Axial, sagittal and coronal sections demonstrate isodose distribution and target coverage for lingual amyloidosis. Clinical target volume (orange), planning target volume (green), parotid glands (yellow) and bone marrow (red) are delineated on CT images for radiation therapy treatment planning. A 5-field coaxial beam arrangement was used for treatment, consisting of 1 anterior-posterior and 4 oblique fields. The planning target volume received a dose of $20 \mathrm{~Gy}$ in 10 fractions.

\section{References}

1 Mardinger O, Rotenberg L, Chaushu G, Taicher S: Surgical management of macroglossia due to primary amyloidosis. Int J Oral Maxillofac Surg 1999;28:129-131.

2 Pepys MB: Amyloidosis. Annu Rev Med 2006;57:223-241.

3 Penner CR, Muller S: Head and neck amyloidosis: a clinicopathologic study of 15 cases. Oral Oncol 2006;42:421-429.

4 Clevens RA, Esclamado RM, DelGaudio JM, Myers MW: Amyloidoma of the neck: case report and review of the literature. Head Neck 1994;16:191-195.

-5 Guijarro-Martínez R, Miragall Alba L, Villar Puchades R, Marqués Mateo M, Puche Torres M, Iglesias Gimilio ME, Pérez-Herrezuelo Hermosa G, Pascual Gil JV: Rational management of macroglossia due to acquired systemic amyloidosis: does surgery play a role? J Oral Maxillofac Surg 2009;67:2013-2017. 
6 Westermark P, Benson MD, Buxbaum JN, Cohen AS, Frangione B, Ikeda S, Masters CL, Merlini G, Saraiva MJ, Sipe JD: Amyloid: toward terminology clarification. Report from the Nomenclature Committee of the International Society of Amyloidosis. Amyloid 2005;12:1-4.

7 Comenzo RL: How I treat amyloidosis. Blood 2009;114:3147-3157.

8 van der Waal RI, van de Scheur MR, Huijgens PC, Starink TM, van der Waal I: Amyloidosis of the tongue as a paraneoplastic marker of plasma cell dyscrasia. Oral Surg Oral Med Oral Pathol Oral Radiol Endod 2002;94:444-447.

9 Fahrner KS, Black CC, Gosselin BJ: Localized amyloidosis of the tongue: a review. Am J Otolaryngol 2004;25:186-189.

10 Kerner MM, Wang MB, Angier G, Calcaterra TC, Ward PH: Amyloidosis of the head and neck. A clinicopathologic study of the UCLA experience, 1955-1991. Arch Otolaryngol Head Neck Surg 1995;121:778782.

11 Prokaeva T, Spencer B, Kaut M, Ozonoff A, Doros G, Connors LH, Skinner M, Seldin DC: Soft tissue, joint, and bone manifestations of AL amyloidosis: clinical presentation, molecular features, and survival. Arthritis Rheum 2007;56:3858-3868.

12 Haraguchi H, Ohashi K, Yamada M, Hasegawa M, Maeda S, Komatsuzaki A: Primary localized nodular tongue amyloidosis associated with Sjögren's syndrome. ORL J Otorhinolaryngol Relat Spec 1997;59:60-63.

13 Nandapalan V, Jones TM, Morar P, Clark AH, Jones AS: Localized amyloidosis of the parotid gland: a case report and review of the localized amyloidosis of the head and neck. Head Neck 1998;20:73-78.

- 14 Palladini G, Perfetti V, Obici L, Caccialanza R, Semino A, Adami F, Cavallero G, Rustichelli R, Virga G, Merlini G: Association of melphalan and high-dose dexamethasone is effective and well tolerated in patients with AL (primary) amyloidosis who are ineligible for stem cell transplantation. Blood 2004;103:2936-2938.

15 Sanchorawala V, Skinner M, Quillen K, Finn KT, Doros G, Seldin DC: Long-term outcome of patients with AL amyloidosis treated with high-dose melphalan and stem-cell transplantation. Blood 2007;110:3561-3563.

-16 Jaccard A, Moreau P, Leblond V, Leleu X, Benboubker L, Hermine O, Recher C, Asli B, Lioure B, Royer B, Jardin F, Bridoux F, Grosbois B, Jaubert J, Piette JC, Ronco P, Quet F, Cogne M, Fernand JP: High-dose melphalan versus melphalan plus dexamethasone for AL amyloidosis. N Engl J Med 2007;357:1083-1093.

17 Sanchorawala V, Seldin DC: An overview of high-dose melphalan and stem cell transplantation in the treatment of AL amyloidosis. Amyloid 2007;14:261-269.

-18 Kurrus JA, Hayes JK, Hoidal JR, Menendez MM, Elstad MR: Radiation therapy for tracheobronchial amyloidosis. Chest 1998;114:1489-1492.

19 Leibovitch I, Selva D, Goldberg RA, Sullivan TJ, Saeed P, Davis G, McCann JD, McNabA, Rootman J: Periocular and orbital amyloidosis: clinical characteristics, management, and outcome. Ophthalmology 2006;113:1657-1664.

20 Neben-Wittich MA, Foote RL, Kalra S: External beam radiation therapy for tracheobronchial amyloidosis. Chest 2007;132:262-267.

21 Capizzi SA, Betancourt E, Prakash UB: Tracheobronchial amyloidosis. Mayo Clin Proc 2000;75:1148-1152.

22 O’Regan A, Fenlon HM, Beamis JF Jr, Steele MP, Skinner M, Berk JL: Tracheobronchial amyloidosis. The Boston University experience from 1984 to 1999. Medicine (Baltimore) 2000;79:69-79.

23 Kalra S, Utz JP, Edell ES, Foote RL: External-beam radiation therapy in the treatment of diffuse tracheobronchial amyloidosis. Mayo Clin Proc 2001;76:853-856.

24 Monroe AT, Walia R, Zlotecki RA, Jantz MA: Tracheobronchial amyloidosis: a case report of successful treatment with external beam radiation therapy. Chest 2004;125:784-789.

25 Gallivan GJ, Gallivan HK: Laryngeal amyloidosis causing hoarseness and airway obstruction. J Voice 2010;24:235-239.

-26 Pasternak S, White VA, Gascoyne RD, Perry SR, Johnson RL, Rootman J: Monoclonal origin of localised orbital amyloidosis detected by molecular analysis. Br J Ophthalmol 1996;80:1013-1017.

-27 Banerjee S, Bogman J, Reuser TT: Amyloid deposition in the extraocular muscles. Orbit 1999;18:105-106

28 Khaira M, Mutamba A, Meligonis G, Rose GE, Plowman PN, O’Donnell H: The use of radiotherapy for the treatment of localized orbital amyloidosis. Orbit 2008;27:432-437.

29 Oluk MA, Murphy J: Nasopharyngeal amyloidosis: an unusual cause for epistaxis. J Laryngol Otol 2010;124:209-212.

30 Poovaneswaran S, Razak AR, Lockman H, Bone M, Pollard K, Mazdai G: Tracheobronchial amyloidosis: utilization of radiotherapy as a treatment modality. Medscape J Med 2008;10:42. 